Special issue of the 2nd International Conference on Computational and Experimental Science and Engineering (ICCESEN 2015)

\title{
Comparison of Reduction Methods for Peak-to-Average-Power-Ratio (PAPR) in MIMO-OFDM Systems with a New Approach
}

\begin{abstract}
M. TÖREN ${ }^{a, *}$ AND C. CIFLIKLI ${ }^{b}$
${ }^{a}$ Department of Electronic and Automation, Vocational School of Technical Science, Recep Tayyip Erdogan University, Rize, Turkey

${ }^{b}$ Department of Electronic and Automation, Vocational High School, Erciyes University, Kayseri, Turkey

This study is based on the comparison of the results of an analysis recommended for the peak-to-averagepower-ratio (PAPR) reduction methods. This is one of the most significant problems in the multiple input-multiple output-orthogonal frequency division multiplexing (MIMO-OFDM) systems. The information in the signals of the wireless communication systems can be given in the frequency domain [S.H. Han, J.H. Lee, IEEE Signal Proc. Lett. 11, 887 (2004)]. Of the methods applied for the PAPR reduction, which is one of the problems occurring in the frequency domain of the signals in MIMO-OFDM systems, the selective mapping (SLM) and the partial transmit sequences (PTS) are compared using a different approach. In the MIMO-OFDM systems, Fourier analysis is performed and the values of the signals on the frequency plane are taken although it is not clear which value of the signal at which time is taken [S.H. Han, J.H. Lee, IEEE Signal Proc. Lett. 11, 887 (2004)]. For the constant state of time, Fourier analysis approach is not appropriate for the detection of accurate frequency values. To solve this problem, a new wavelet transform-based (WT) analysis is recommended for the MIMO-OFDM systems with a new approach. It is shown that this analysis will be preferred instead of the Fourier analysis because of the optimization of the frequency-based temporary solutions of the signal. This approach of analysis is used along with the methods used for solving the PAPR problem in the MIMO-OFDM systems. Thereby, a comparison showing at which rate the methods for the solution of the problem contribute to solving the problem with this approach can be carried out. Numerical results show at which rate the methods with this approach can be carried out to contribute for solving the problem.
\end{abstract}

DOI: 10.12693/APhysPolA.130.417

PACS/topics: 84.40.Ua

\section{Introduction}

In recent years, a recommended method for meeting the requirements of the wireless communication systems in frequency-selective fading channels has been the orthogonal frequency division multiplexing (OFDM) system [1]. The necessary requirements are secure communication, bandwidth efficiency, high data rate, etc. according to the location of the signals. Advantages such as high data transmission rate, bandwidth saving and reliability are inherent to the OFDM systems. Moreover, the OFDM system effectively provides numerous parallel narrow band channels and is used along with the multiple input-multiple output (MIMO) systems, which in turn increases the data transmission rate, diversity gain and system capacity $[2,3]$. MIMO-OFDM systems are regarded as key technology for wireless communication systems with high data rate in the current communication systems and are used in digital subcarrier lines (DSL), IEEE802.11, IEEE802.16, IEEE 802.15.3a and satellite connections in 4G technology [4].

Despite it being a key technology, there are problems in MIMO-OFDM systems. The peak-to-average-power- ratio (PAPR) problem is one of the most significant of these problems and there are several recommended methods for solving it [5-7]. These methods are divided in two groups. The first group includes signal jamming methods (clipping, peak windowing, etc.). Of these methods, the clipping method is the simplest one for the PAPR reduction. However, the method jams the signal due to the out-of-band emission and interference [8].

Signal interference methods (code-based block coding, stochastic selective mapping and partial transmit sequences) are available in the second group. In these methods, while PAPR is reduced in the block coding method, the data rate is also reduced [9] and the signal energy is increased. Selective mapping and partial transmit sequences, which are the stochastic methods in this group, do not cause any jamming in the signals or increasing in the signal energy.

In this study, analysis is performed by examining the lower block number and subcarrier parameters important in the selective mapping (SLM) method and reduction in the PAPR rate is obtained for two parameters; again in the partial transmit sequences (PTS) method analysis is performed for the lower block number and different phase parameters, and the results are compared. 


\section{MIMO-OFDM system and PAPR}

The general structure which constitutes the system consists of the multiplexing system, MIMO and OFDM system where this multiplexing is modulated.

Diversity, which is acquired by spatial decomposition of the antennas in the multipath scattering environments, is available in MIMO. MIMO systems accommodate antenna arrays in the transmitter and the receiver. The purpose of this is based on the suggestion that the signals be detected by multiple receivers during the proper transferring and receiving thereof by the respective side $[10,11]$.

The aim of combined MIMO system with OFDM modulation is to increase the efficiency. The block diagram of the system is shown in Fig. 1.

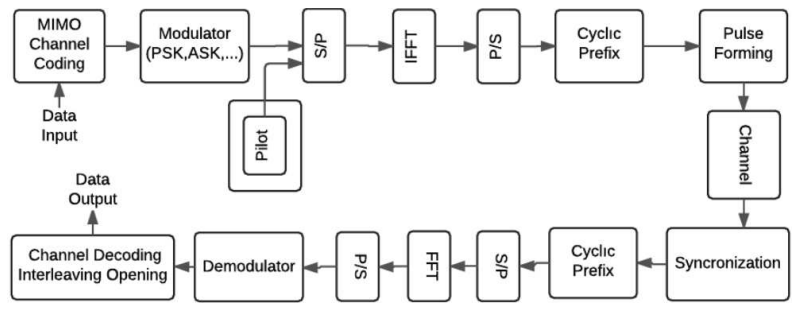

Fig. 1. MIMO-OFDM block diagram.

If the signals, which will be transferred, in OFDM are identified, $K$ subcarrier data block in the $N$-length data block is formed in the following way

$$
X_{k}=\left(X_{0}, X_{1}, \ldots, X_{N-1}\right) \text {, }
$$

in a manner to correspond to all of the subcarriers [2].

Each $X_{k}$ signal is modulated in frequency values between $f_{k}(k=0, \ldots, N-1)$ subcarriers, if $f_{k}=k \Delta f, N$ sub-data blocks are orthogonal. Here, $f_{k}=\frac{1}{T}$ and $T$ are OFDM signal periods [12].

$X$ is a complex signal vector, transmitted in the form of $x=\left[x_{0}, x_{1}, \ldots, x_{N-1}\right]$ as a discrete time signal with the inverse discrete fast Fourier (IDFT) transformation in the frequency plane.

$$
x=\operatorname{IDFT}(X) \text {. }
$$

Due to the fact that all subcarriers of the signals transmitted are statistically independent, the signal samples are complex Gaussian distributed and are of highamplitude in the time plane [12].

High amplitude value occurring in OFDM system creates PAPR which causes a disadvantage in the system. In OFDM system, PAPR value of the transmitted signal $x(t)$ is defined as follows:

$$
\operatorname{PAPR}\{x(t)\}=\frac{\max |x(t)|^{2}}{E\left\{\left|x(t)^{2}\right|\right\}},
$$

were $E$ is the expectation operator.

Here, when PAPR expressions of the multiple inputmultiple output systems are discussed with respect to the single input-single output systems, in the studies in literature it is observed that multiple input-multiple output systems are of higher performance [12].

\section{PAPR reduction methods}

\subsection{Selective mapping (SLM) and partial transmit sequence (PTS) methods}

The methods used to the reduce PAPR value in MIMO-OFDM systems can be examined by dividing them into two groups; signal jamming methods (clipping, peak windowing, etc.) and signal interference methods (such as selective mapping (SLM), partial transmit sequence (PTS), block coding method, etc.) respectively.

SLM [13, 14], transmitter generates different data block candidates at a sufficient number, each of which resembles the original data block, and selects out of them the one with the lowest PAPR value $[15,16]$.

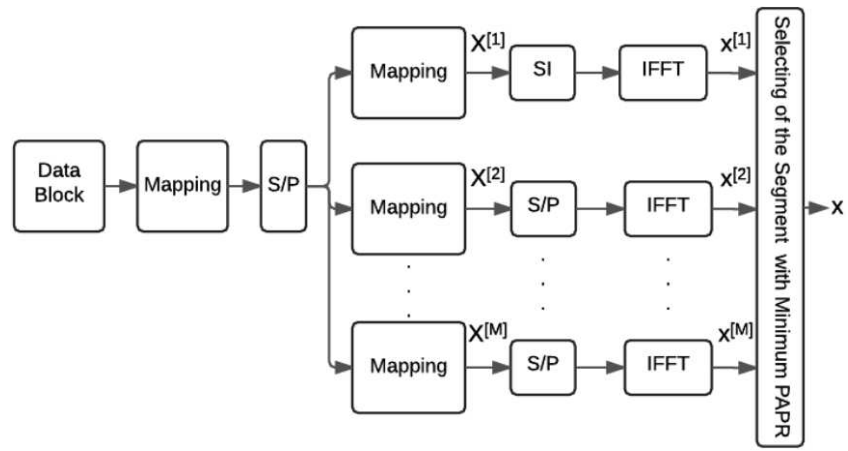

Fig. 2. SLM method block diagram.

The block diagram of the SLM method is seen in Fig. 2. Each of $N$ sub-data carriers is multiplied by different $M$ independent data blocks.

The PTS method [17, 18] was first recommended by S.H.Müller and J.B.Huber [19]. With PTS, $N$ data input block signals are divided into separate sub-blocks. The subcarrier of each sub-block is weighted with phase factor for the sub-block. The phase factor is selected in a manner to minimize the PAPR value of the total signal. The block diagram of the PTS method is given in Fig. 3.

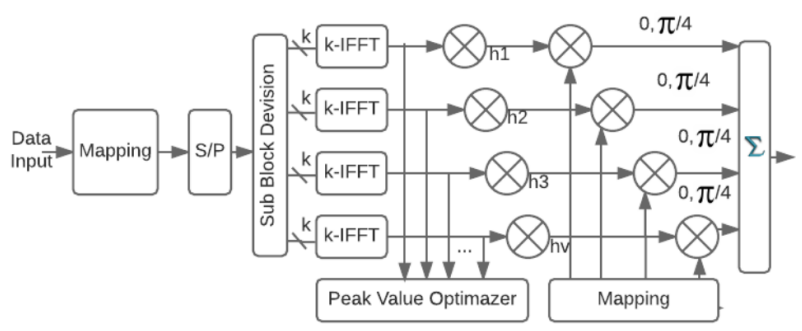

Fig. 3. PTS method block diagram.

As will be understood from the descriptions herein for the method used in order to reduce PAPR, the reduction is based on the $V$ sub-block number and $W$ allowed phase factor $b$-number. 


\section{Wavelet transform (WT)}

Wavelet transform provides analysis of non-stationary signals. The time-frequency resolution is optimally obtained. Wavelet transform and analysis are generalized form of the Fourier analysis. Such analysis is preferred in this study instead of the Fourier transform (FT) block. This is because the FT block does not yield the desired result in temporary situations when it is used for the signals that do not vary according to time. To solve this problem the short Fourier transform (SFT) has been developed. However, as this analysis cannot detect the accurate frequency, time wavelet transform serves as an alternative to this transform. Wavelet transform generates results for the signals in both the time and the frequency plane.

In the wavelet theory, the representation of the wavelet and scaling functions are provided by the coefficients of high pass filter $h[m]$ and low pass filter $g[m]$. High pass filters are related to wavelet function while low pass filters are related to the scaling function. While being received in the receiver section, the signal passes through these filters. Therefore, the wavelet transform can be applied easily to use with discrete-time filters [2]. The mathematical expression of wavelet transform is as follows

$$
\begin{aligned}
& \psi_{k+1,2 p-1[m]}=\sqrt{2} \sum_{m^{\prime}} h\left[m^{\prime}\right] \psi_{k, p\left[m-2^{k} m^{\prime}\right]}, \\
& \psi_{k+1,2 p[m]}=\sqrt{2} \sum_{m^{\prime}} g\left[m^{\prime}\right] \psi_{k, p\left[m-2^{k} m^{\prime}\right]},
\end{aligned}
$$

wherein $\psi_{k, p[m]}$ is the $p^{\text {th }}$ wavelet transform function on $k^{\text {th }}$ level [20].

When the signal is transmitted with IDWT instead of IFFT, it is defined as follows

$$
\begin{aligned}
& x=\operatorname{IDWT}(X), \\
& x(t)=\sum_{p=0}^{N=1} \sum_{l=0}^{\infty} X(l) \psi_{k, p}^{\mathrm{sym}}(t-l N),
\end{aligned}
$$

wherein, $p$ is $l$ the location data index, $\psi_{k, p}^{\mathrm{sym}}$ is the wavelet packet function for the $p$ sub-channel, and $X(l)$ are the data signals. at different $\mathrm{W}$ (different phase) values when WT is incorporated into MIMO-OFDM system using PTS method

\section{Simulation results}

The simulation which was performed in this study has removed the FT section of MIMO-OFDM system and incorporated the WT section, before the analysis. Likewise, in the receiver system, WT section is used instead of FT section and then the analysis is made. MIMO-OFDM model used in simulation is shown in Fig. 4. Different values of the variable parameters used in the mathematical expressions of investigated methods are summarized in Table. The results are shown in Figs. 5-9.

\section{Conclusions}

The SLM and PTS methods of PAPR reduction methods are studied. These are commonly used for reducing

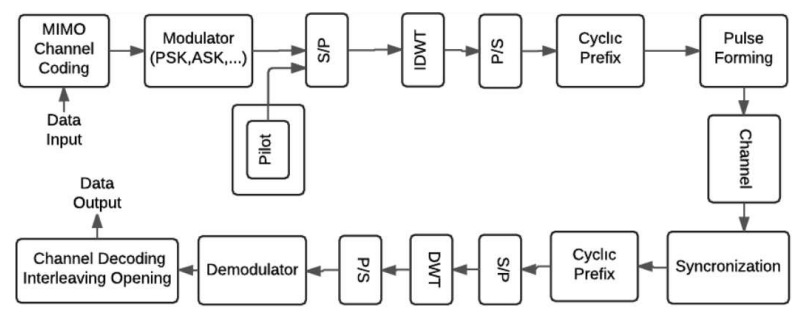

Fig. 4. MIMO-OFDM model used in simulation.

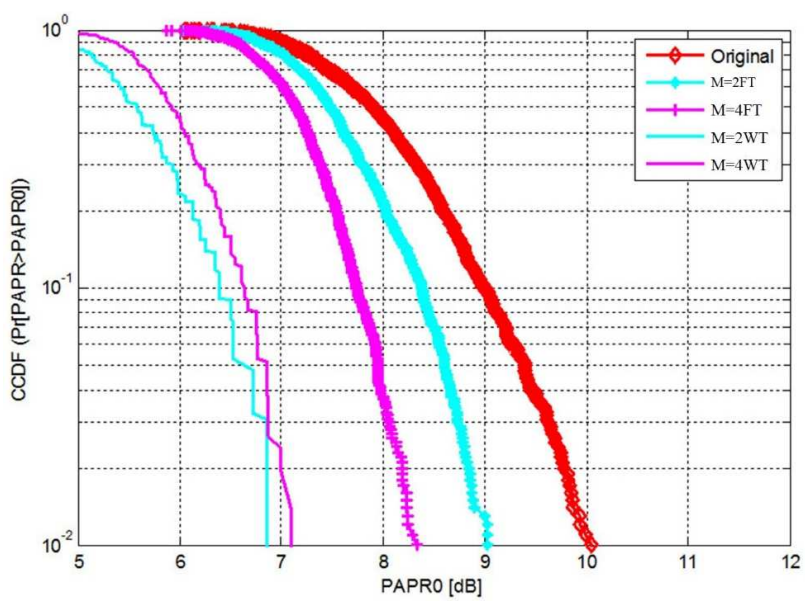

Fig. 5. PAPR reduction graph at different $M$ values $(M=2,4)$ when WT is incorporated into MIMOOFDM system using SLM method.

PAPR, which is a disadvantage on the signal transmitted in the MIMO-OFDM system. According to the different values of the variable parameters in the mathematical expressions of these methods, which are important for PAPR reduction, FT and WT analysis is performed and the percentage changes of the values are obtained. It is observed from the changes in percentages that the rate of the SLM method and the rate of the PTS method

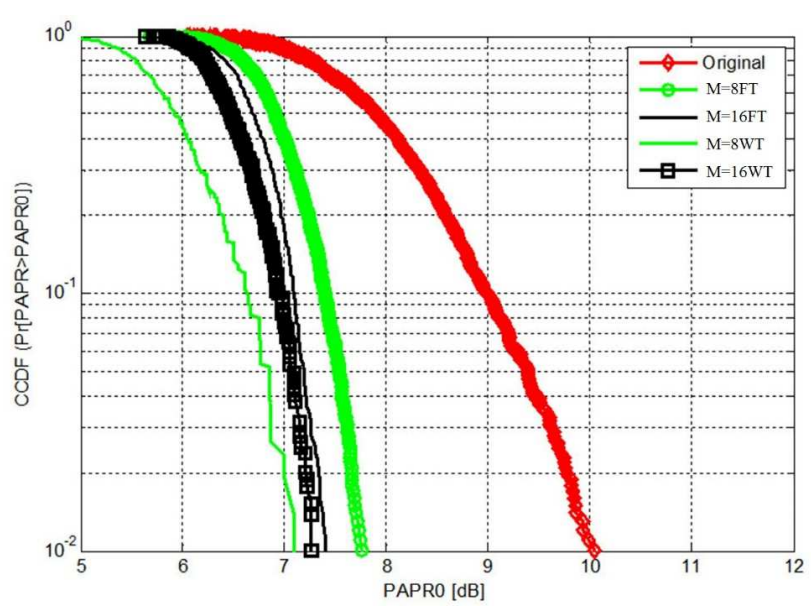

Fig. 6. PAPR reduction graph at different $M$ values $(M=8,16)$ when WT is incorporated into MIMOOFDM system using SLM method. 


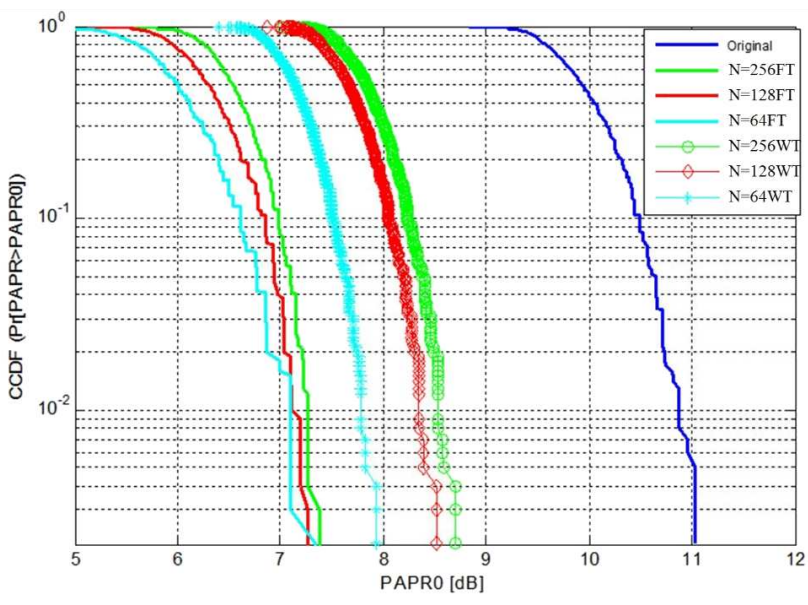

Fig. 7. PAPR reduction graph at different $N$ values $(N=64,128,256)$ when WT is incorporated into MIMO-OFDM system using SLM method.

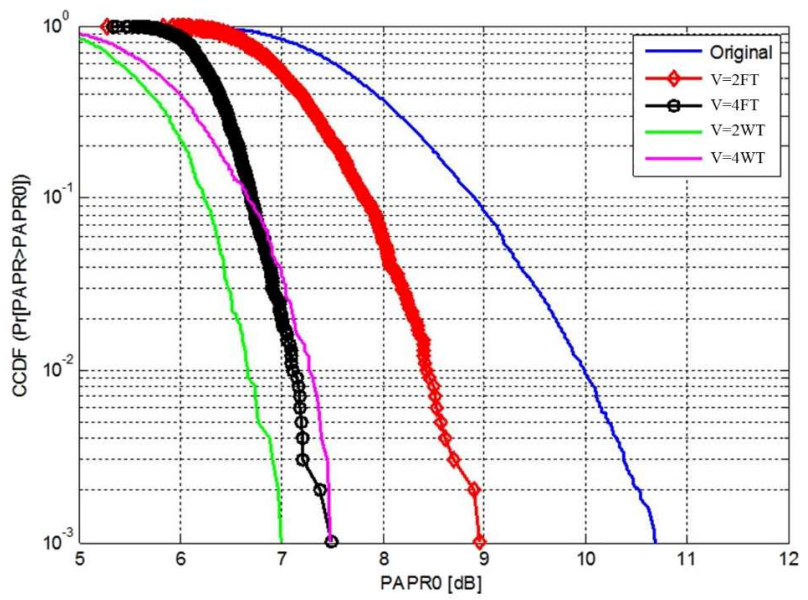

Fig. 8. PAPR reduction graph at different $V$ (subblock number) values when WT is incorporated into MIMO-OFDM system using PTS method.

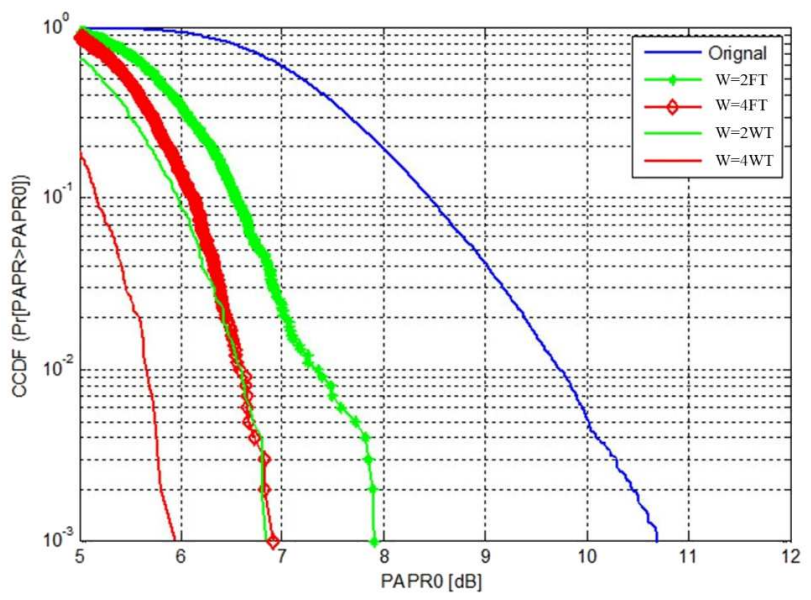

Fig. 9. PAPR reduction graph. have yielded low values for PAPR reduction and with WT analysis these values have dropped down even more.

\section{References}

[1] S.H. Han, J.H. Lee, IEEE Signal Proc. Lett. 11, 887 (2004).

[2] D. Gesbert, M. Shafi, D. Shiu, P.J. Smith, A. Naguib, IEEE J. Sel. Area Comm. 21, 281 (2003).

[3] Z.F. Li, X.G. Xia, IEEE Trans. Wireless Comm. 7, 1195 (2008).

[4] P.D.M. Pandurangan, D. Perumal, (IJCSI) Int. J. Comput. Sci. Iss. 8, 444 (2011).

[5] V.C. Ramasami, KUID Report 688659, 1 (2001).

[6] A.F. Molisch, M. Steinbauer, M. Toeltsch, E. Bonek, R.S. Thomä, IEEE J. Sel. Area Comm. 20, 561 (2002).

[7] X.D. Li, L.J. Cimini, IEEE Signal Proc. Lett. 2, 131 (1998).

[8] V. Tarokh, H. Jafarkhani, IEEE T. Commun. 48, 37 (2000).

[9] J. Fitzpatrick, Simulation of a Multiple Input Multiple Output (MIMO) Wireless System, Dublin City University, 2004, p. 3.

[10] R.W. Bauml, R.F.H. Fischer, J.B. Huber, Electron. Lett. 32, 2056 (1996).

[11] S.H. Muller, J.B. Huber, in: Globecom 97-IEEE Global Telecommunications Conference, Conference Record, Vols. 1-3, 1997, p. 1.

[12] B. Rihawi, Y. Louet, S. Zabre, in: l.J. Commun. Network System Sci. 1, 29 (2008).

[13] M. Breiling, S.H. Muller-Weinfurtner, J.B. Huber, IEEE Commun. Lett. 5, 239 (2001).

[14] S.Y. Cho, K.J. Yang Y.W. Kang, MIMO-OFDM Wireless Communications with MATLAB, 2010.

[15] S.H.Han, J.H. Lee, IEEE Trans. Wireless Comm. 12, 56 (2005).

[16] P.S.M. Chauhan, H. Patel, Int. J. Eng. Res. Apl. (IJERA) 2, 1292 (2012).

[17] S.H. Muller, J.B. Huber, Pimrc'97 - Eighth IEEE International Symposium on Personal, Indoor and Mobile Radio Communications: Waves of the Year 2000+, Vols. 1-3, 1997, p. 1090.

[18] A.H.M. Shahparan, Numerical Performance Evaluation of PAPR Reduction Tecniques in MIMO-OFDM Wireless Communication System, Noakhali and Technology University, 2010

[19] S.H.Muller, J.B. Huber, Elect. Lett. 33, 368 (1997).

[20] M. Baro, J. Ilow, in: 2008 5th IEEE Consumer Communications and Networking Conference, Vols. 1-3, 2008, p. 195. 
TABLE

Values of SLM and PTS methods in MIMO-OFDM system in FT and WT analysis and change in \%.

\begin{tabular}{c|c|c|c|c|c|c|c|c|c|c}
\hline \hline & \multicolumn{3}{|c|}{ Fourier transform values } & \multicolumn{3}{c|}{ Wavelet transform values } & \multicolumn{2}{c}{$\begin{array}{c}\text { Ratio of } \\
\text { percentage change }\end{array}$} \\
\hline \multirow{4}{*}{ SLM } & $\mathrm{M}$ & $\mathrm{M}$ & $\mathrm{N}$ & $\mathrm{N}$ & $\mathrm{M}$ & $\mathrm{M}$ & $\mathrm{N}$ & $\mathrm{N}$ & $\mathrm{M}$ & $\mathrm{N}$ \\
& Values & PAPR & Value & PAPR & Values & PAPR & Value & PAPR & $\%$ & $\%$ \\
\hline & 2 & 9.1 & 64 & 8.2 & 2 & 6.85 & 64 & 7.3 & 24.73 & 10.98 \\
& 4 & 8.4 & 128 & 8.7 & 4 & 7.6 & 128 & 7.2 & 9.52 & 17.24 \\
& 8 & 7.8 & 256 & 9 & 8 & 7.1 & 256 & 7.4 & 8.97 & 17.78 \\
& 16 & 7.4 & - & - & 16 & 7.3 & - & - & 1.35 & - \\
\hline \multirow{5}{*}{ PTS } & $\mathrm{V}$ & $\mathrm{V}$ & $\mathrm{W}$ & $\mathrm{W}$ & $\mathrm{V}$ & $\mathrm{V}$ & $\mathrm{W}$ & $\mathrm{W}$ & $\mathrm{V}$ & $\mathrm{W}$ \\
& Value & PAPR & Value & PAPR & Value & PAPR & Value & PAPR & $\%$ & $\%$ \\
\cline { 2 - 10 } & 2 & 8.9 & 2 & 7.9 & 2 & 7.5 & 2 & 6.85 & 15.73 & 13.29 \\
& 4 & 7.5 & 4 & 6.9 & 4 & 7 & 4 & 5.9 & 6.67 & 14.49
\end{tabular}

